\title{
Studies of defects in the near-surface region and at interfaces using low energy positron beams
}

\author{
P ASOKA-KUMAR \\ Department of Physics, Brookhaven National Laboratory, Upton, New York 11973, USA
}

\begin{abstract}
Positron annihilation spectroscopy (PAS) is a powerful probe to study openvolume defects in solids. Its success is due to the propensity of positrons to seek out low-density regions of a solid, such as vacancies and voids, and the emissions of gamma rays from their annihilations that carry information about the local electronic environment. The development of low-energy positron beams allows probing of defects to depths of few microns, and can successfully characterize defects in the near-surface and interface regions of several technologically important systems. This review focuses on recent studies conducted on semiconductorbased systems.
\end{abstract}

Keywords. Defects; positron beams.

\section{Introduction}

The last three decades saw an explosive growth in methods to produce materials with unique properties. Many of these techniques allowed the engineering of high-quality layered structures (both homo- and hetero-structures). Along with these advances, the need to characterize the defect content of these structures became evident. With many advanced materials, a single characterization method often is inadequate. Therefore, the material-research community has relied on a variety of techniques to control and optimize their processing steps. In this context, positron annihilation spectroscopy (PAS) has become a powerful, nondestructive probe to characterize dilute quantities of open-volume defects.

With the development of intense, variable-energy positron beams, it is possible now to examine defects in the near-surface regions and buried interfaces. Below, we describe the application of PAS to several advanced semiconductor-based systems, and include the following topics: defects in MBE-grown $\mathrm{Si}$ and $\mathrm{GaAs}$, hydrogen interaction with $\mathrm{Si}$ dangling bonds, and electromigration-induced vacancy formation.

\section{Positron annihilation spectroscopy}

We first review the technique, emphasizing recent progress. PAS, as the name suggests, is the spectroscopy of photons emerging from the annihilation of positrons with electrons. The depth-resolved PAS method is based on: (i) the availability of beams (Brandt and Dupasquier 1983; Vehanen 1984; Reichel and Yelon 1985; Coleman and Walker 1986; Schultz and Lynn 1988; Schultz et al 1990; Ottewitte and Weiss 1994) of positrons that can be used to probe various controlled depths (in the range of a few $\mu \mathrm{m}$ ) (Trifthäuser and Köegel 1982) of a material; (ii) the propensity of positrons to seek out low-density regions of a solid such as voids and vacancies (Berko and Erskine 1967; MacKenzie et al 1967) and (iii) the annihilation of positrons and electrons into $\gamma$-rays that carry information about the low-ion-density regions and escape the test material 
without significant attenuation (Brandt and Dupasquier 1983). In bulk materials, the technique can detect vacancy-like defects at a sensitivity level of $5 \times 10^{15} \mathrm{~cm}^{-3}$.

When an energetic positron enters a material, it thermalizes rapidly (within 1-10 psec) and undergoes diffusion (or is trapped at a defect site) until it annihilates with an electron. The thermalized positron experiences a potential with large repulsive spikes centred on the ion cores. A defect site with a missing atom(s) offers a local energy minimum for positrons. Consequently, open-volume-type defects are easily seen by positrons. The annihilation photons carry information about the centre-of-mass motion of the electron-positron pair, and because the positrons are thermalized, this information essentially is dominated by the electron momentum. The information derived from the photons leads to several observables: lifetime, angular correlation, and Doppler shift (Brandt and Dupasquier 1983). In this review, we will confine ourselves to the last one, because most of the depth-resolved PAS was achieved by measuring Doppler shift.

Annihilation of a positron-electron pair produces photons with a total energy of $2 m_{0} c^{2}$ minus the electron binding energy, where $m_{0} c^{2}(=511 \mathrm{keV})$ denotes the electron's (positron's) rest-mass energy. Without a third body participating in the annihilation process (Palthingal et al 1991), the energy-momentum conservation demands the emission of two or more photons, with two $\gamma$-channel dominating in most situations*. The centre-of-mass motion of the annihilating pair (dominated by the electron momentum) causes the photons to be Doppler-shifted from the centre energy of $511 \mathrm{keV}$. In an experiment recording the energies of several annihilation photons (both up- and down-shifted), the Doppler shift of the individual $\gamma$-ray will contribute to an overall broadening of the annihilation photopeak; this often is called Doppler broadening.

The Doppler-broadened spectrum can be characterized by using some part of it; one such parameter is the $S$ parameter, defined as the ratio of counts (i.e. area) in a central region of the photopeak to the total counts (area) in the peak. The S-parameter has a simple relationship to Doppler broadening: For example, when positrons annihilate predominantly with slow-moving electrons, Doppler broadening is small and $\mathrm{S}$ parameter is large, and vice versa. Similarly, by taking the ratio with the wing region instead of the central region yields the W-parameter. Thus, annihilations with low-momentum electrons are reflected in the $\mathrm{S}$ parameter and those with high-momentum electrons in the $\mathrm{W}$ parameter. At open-volume-defect sites, positrons annihilate predominantly with low-momentum electrons as opposed to normal material where positrons are likely to encounter highmomentum core electrons. As a result, open-volume defects produce a high $\mathrm{S}$ parameter. More often, $\mathrm{S}$ and $\mathrm{W}$ parameters show opposite behaviour.

The use of simple parameters, such as $S$ and $W$, yield extensive information about the defect content of a material. The absolute values of these parameters are of little significance, since they are decided by the width of the window region chosen to define them. Therefore, these parameters are normalized to a reference value corresponding to a defect-free material (as seen by positions). The normalized values then can be compared between different samples and different experimental arrangements. Because the measurements of $S$ (or $W$ ) versus positron energy can be performed and interpreted very rapidly, they are used extensively in depth-resolved PAS studies.

The Doppler broadening studies can be extended further to identify impurityvacancy binding (MacDonald et al 1978; Alatalo et al 1995; Szpala et al 1995).

\footnotetext{
*Three- $\gamma$-decay mode is visible during positronium (a bound state of positron and electron) decay and is used to study external and internal surfaces.
} 
Lynn $e t$ al showed how the annihilations of positrons with inner shell (core) electrons can be examined using a coincidence system. Instead of detecting a single $\gamma$-ray from each annihilation, the technique detects both $\gamma$-rays in coincidence. The coincidence suppresses the background and allows examinations of annihilations with inner shell electrons, which contribute mainly to the tail of the Doppler-broadened spectrum. Because inner shell electrons retain their properties even when the atoms are embedded in a host lattice, this approach can be used to identify impurity atoms.

\section{Applications}

\subsection{Defects in MBE grown Si and GaAs}

Molecular beam epitaxy (MBE) of semiconducting materials allows engineering of device structures with tailored electrical properties. In many instances, lowering the growth temperature to prevent unwanted segregation and diffusion of the dopants is desirable. In $\mathrm{Si}$, at conventional $\mathrm{MBE}$ growth temperatures $\left(\geqslant 450^{\circ} \mathrm{C}\right)$, dopant segregation is severe and its incorporation is below unity (This is the so called "Si doping problem"). A lower growth temperature will solve both problems, but the quality of the deposited film is expected to be poor. In a pioneering work, Eaglesham et al (1990) and Gossmann et al (1990) showed that epitaxy can be sustained even at room temperatures to a critical thickness $h_{\text {epi }}$, beyond which crystalline growth becomes amorphous. $h_{\text {epi }}$ increases with increasing growth temperature, and the memory of the limiting epitaxial thickness can be erased by a rapid thermal anneal (RTA), in which the growing film receives a short $(150 \mathrm{sec})$ exposure to elevated temperature $\left(T_{\mathrm{RTA}} \sim 500^{\circ} \mathrm{C}\right)$.

The low temperature employed during MBE growth raises the question of the quality of the film. Conventional techniques, such as electron microscopy, rule out extended defects and ion-scattering places a high limit on point-defect concentrations $\left(\sim 10^{18} \mathrm{~cm}^{-3}\right)$. On the other hand, PAS, with a simple $\mathrm{S}$ parameter vs energy measurement, offers a lower limit $\left(\sim 5 \times 10^{15} \mathrm{~cm}^{-3}\right)$ to vacancy-like defects in crystalline materials. Detailed studies on these class of materials showed the unique capabilities of the positron probe in solving defect-related problems (Gossmann et al 1992; Asoka-Kumar et al 1993, 1995: Szeles et al 1995; Szpala et al 1995).

Figure 1 shows S-parameter measurements from an MBE-grown Si/Si structure as a function of depth into the sample. Gossmann et al (1992) give the details of the growth process. The figure compares results from two samples grown at $220^{\circ} \mathrm{C}$ with different RTA temperatures. For comparison, we include the result from a commercial Si wafer. Ion-scattering and electron microscopy measurements show that films grown with the RTA at $600^{\circ} \mathrm{C}$ and $450^{\circ} \mathrm{C}$ are indistinguishable. However, positron measurements show a clear difference between them. The sample grown at $T_{\text {RTA }}=600^{\circ} \mathrm{C}$ is identical to control $\mathrm{Si}$, thus setting an upper limit of $5 \times 10^{15} \mathrm{~cm}^{-3}$ for vacancy-like defects. There is a sharp increase in the defect signal (indicated by a high S-value (Asoka-Kumar et al 1994a)) when the RTA temperature is lowered to $450^{\circ} \mathrm{C}$. Analysis of the S-parameter results yield a defect concentration of $\sim 10^{18} \mathrm{~cm}^{-3}$ (Asoka-Kumar et al 1993). The transition in the film properties with $T_{\mathrm{RTA}}$ was explained in terms of the hydrogen incorporated in the growing film at a lower RTA temperature (AsokaKumar et al 1995). 


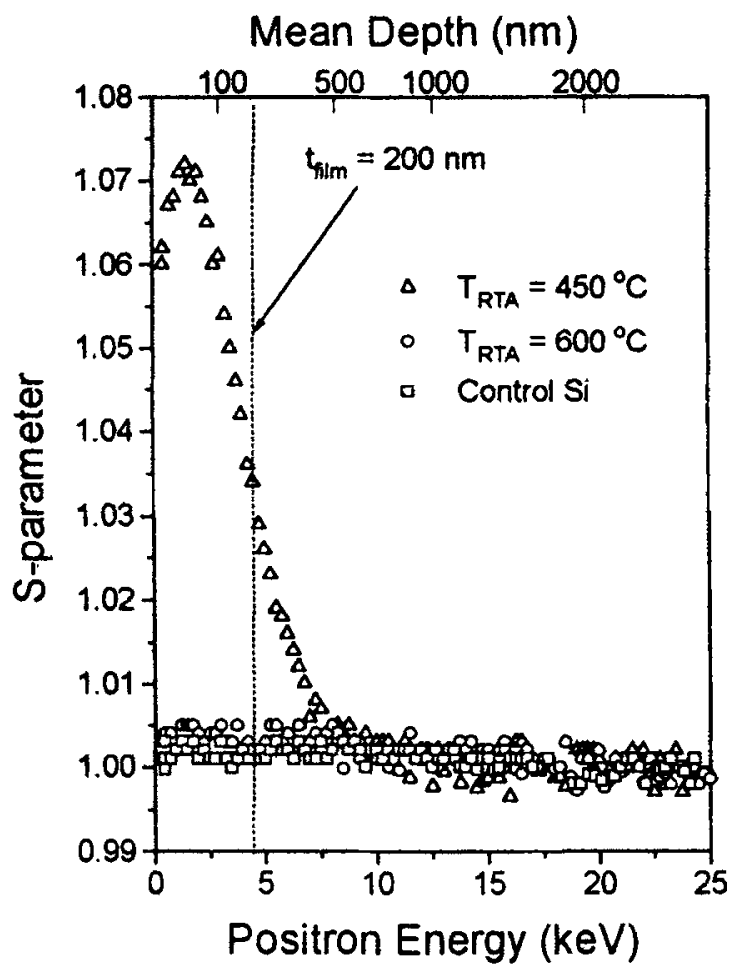

Figure 1. Depth-resolved S-parameter results from MBE samples grown at $220^{\circ} \mathrm{C}$ with different RTA temperatures. Result from a control Si sample also is shown.

The importance of the defect layer detected using PAS in samples grown with $T_{\mathrm{RTA}}=450^{\circ} \mathrm{C}$ was realized when electrical characteristics of pn junctions made from these materials were examined (Asoka-Kumar et al 1995). The current-voltage ( $I-V)$ characteristics of two batches of samples showed that diodes made from films with $T_{\mathrm{RTA}}=600^{\circ} \mathrm{C}$ have significantly smaller reverse-bias leakage currents than those with $T_{\mathrm{RTA}}=450^{\circ} \mathrm{C}$, revealing that there are defects in the latter, consistent with the PAS study. Also, the $I-V$ characteristics of the diode made from films with $T_{\mathrm{RTA}}=450^{\circ} \mathrm{C}$ can be modeled successfully by including the defect concentration $\left(10^{18} \mathrm{~cm}^{-3}\right)$ derived from the positron measurements.

As stated earlier, the attractiveness of low-temperature MBE is that it solves the $\mathrm{Si}$ doping problem so that films can be doped to high concentrations to produce novel device structures. At low doping concentrations, the carrier concentration increases linearly with the number of dopant atoms, suggesting that all dopant atoms are activated. But at high concentrations, this picture breaks down, and the mechanism for the deactivation is not fully understood. In Sb-doped Si, the unit activation can be maintained up to $\sim 7 \times 10^{20} \mathrm{~cm}^{-3}$, beyond which increasing the $\mathrm{Sb}$ concentration does not produce a corresponding increase in the carrier concentration.

The defect responsible for the compensation process was examined with a newly developed coincidence system (Szpala et al 1995). By reducing the background and examining the tail region of the Doppler-broadened spectrum, the $\mathrm{Sb}$ atoms were shown to be tied with vacancies at high doping concentrations. These results showed 
that chemical variations in the near-neighbour occupancy of an open-volume defect can be demonstrated with PAS.

GaAs layers grown by low-temperature MBE offer many unique properties. They have been used as an insulating buffer layer for GaAs devices, and as a material for subpicosecond switches. Their insulating property is achieved only after annealing the material at $\sim 600^{\circ} \mathrm{C}$, upon which, resistivity increased by several orders of magnitude; the mechanism responsible for this behaviour is not fully understood. Different authors used models based on arsenic clusters and different point-defects. PAS measurements show a significant increase in vacancy concentration upon annealing (Keeble et al 1993; Umlor et al 1995). The anneal-induced defect formation occurs concurrently with increases in resistivity and lattice relaxation.

\section{Hydrogen interaction with $\mathrm{Si}$ voids and $\mathrm{SiO}_{2}-\mathrm{Si}$ interface}

Hydrogen is a common element found in a device-processing ambient and plays an important role in many device characteristics. In particular, hydrogen is present simultaneously with the generation of defects in plasma-processing environments, inducing complex reactions and passivation mechanisms in the near-surface region. PAS studies of Si subjected to an rf hydrogen-plasma revealed microvoids in the near-surface region and showed passivation of the voids' surface with hydrogen (Asoka-Kumar et al 1994b). These microvoids are stable to at least $800^{\circ} \mathrm{C}$ and would, therefore, be retained through low-temperature processing steps. The hydrogen coverage of the void surface can be examined by changes in the positron signal. A high temperature $\left(>600^{\circ} \mathrm{C}\right)$ vacuum anneal removed hydrogen from the internal surfaces; it

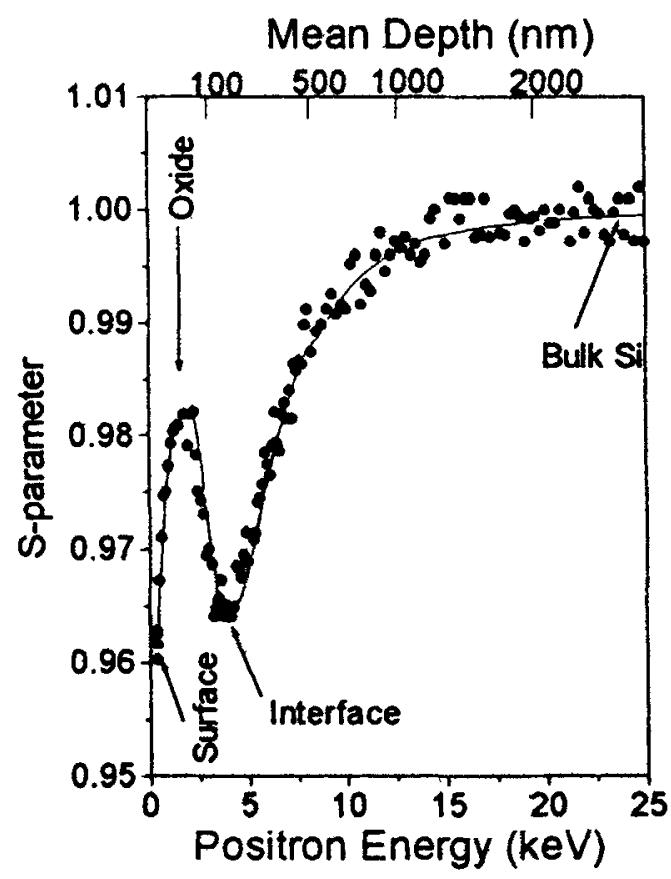

Figure 2. The S-parameter results from a $\mathrm{SiO}_{2}-\mathrm{Si}$ system showing an interface signal. 
can be reloaded by annealing in a hydrogen ambient. Positron measurements showed a corresponding reversible change in the $S$-parameter. While this study was restricted to hydrogen, the possibility of using positron annihilation to investigate gettering and the interactions of other impurities with void surfaces is obvious.

In metal-oxide-semiconductor technology, hydrogen passivation of the dangling bond sites at $\mathrm{SiO}_{2}-\mathrm{Si}$ interfaces has long been used to reduce the density of electrically active interface trap sites. PAS studies conducted on a $\mathrm{SiO}_{2}-\mathrm{Si}$ system revealed the kinetics of hydrogen interaction with these trap sites. The recent surge in studies of $\mathrm{SiO}_{2}-\mathrm{Si}$ using positrons started with the realization that positron measurements show an interface signal that is distinct from bulk oxide and $\mathrm{Si}$ (see figure 2) (Nielsen et al 1987).

The interface S-parameter is sensitive to the passivation state of the interface traps (Khatri et al 1994). Figure 3 shows the behaviour of the interface S-parameter as a function of annealing temperature carried out for $30 \mathrm{~min}$, each in steps of $50^{\circ} \mathrm{C}$. Before the start of each step, the sample was annealed in a hydrogen ambient at $400^{\circ} \mathrm{C}$ for 30 min to produce an interface with maximum number of hydrogen-passivated dangling bond sites (Nicollian and Brews 1982). Upon annealing, the interface S-parameter increases in proportion to the number of thermally broken Si-H bonds (figure 3). This increase is completely reversible, and the original $\mathrm{S}$-value can be regained by annealing the sample again in a hydrogen ambient. Assuming a first-order process for the thermal breakup of $\mathrm{Si}-\mathrm{H}$ bonds, the activation energy for breaking an $\mathrm{Si}-\mathrm{H}$ bond is $2.60 \pm 0.6 \mathrm{eV}$. The importance of these results arises from the fact that they were obtained with a nondestructive probe, and did not require any special preparation of the sample.

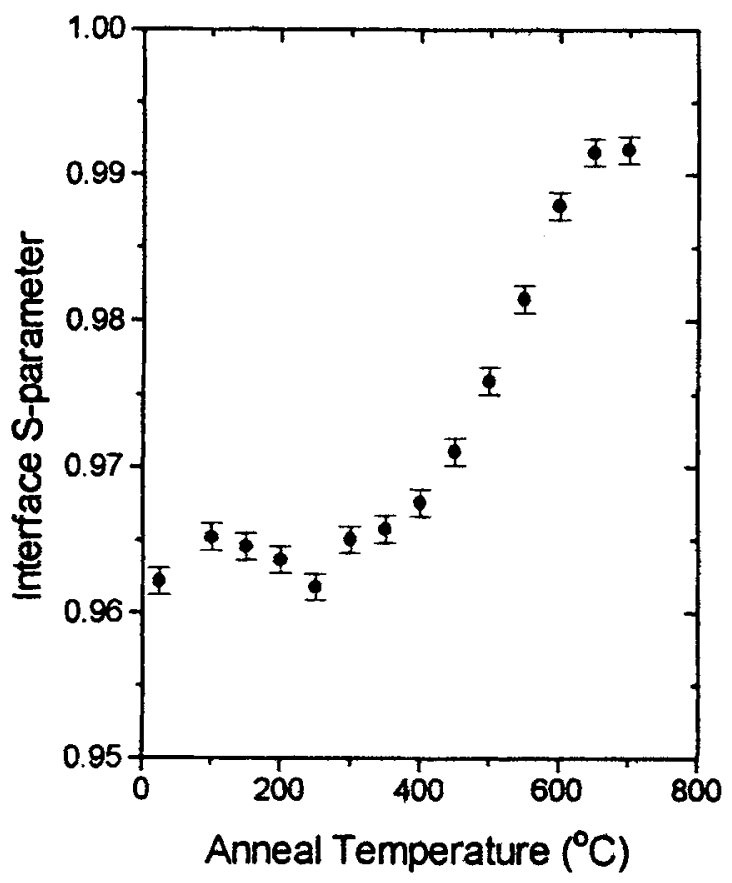

Figure 3. Annealing behaviour of the interface S-parameter of an $\mathrm{SiO}_{2}-\mathrm{Si}$ system. 


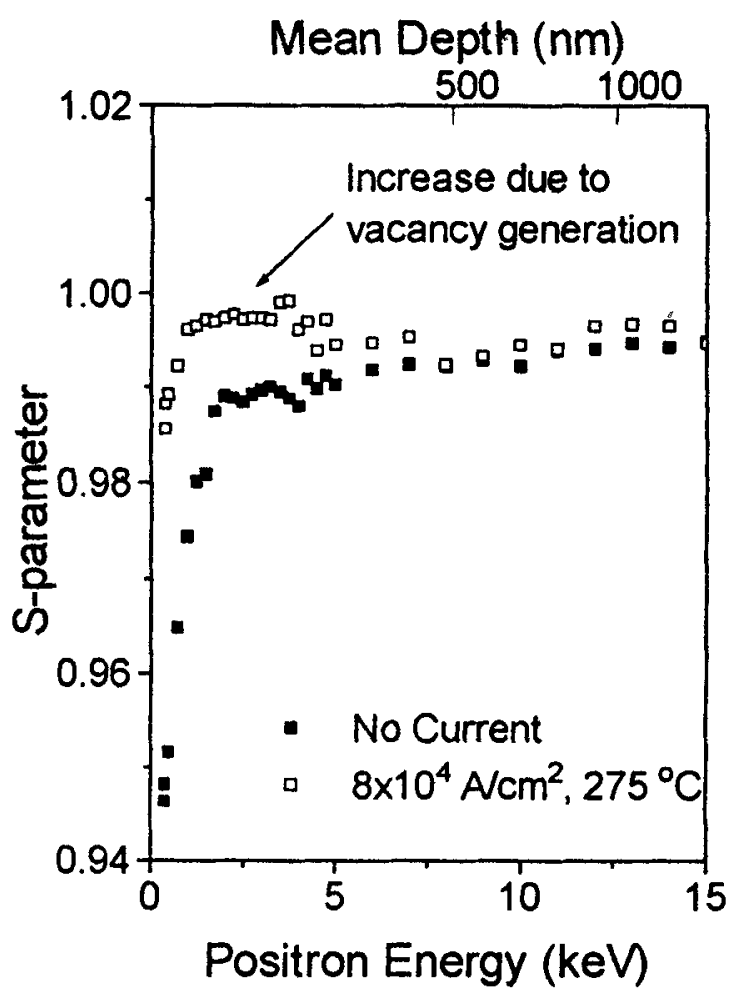

Figure 4. S-parameter measurements from a system containing $\mathrm{Al}(\mathrm{Cu})$ lines on $\mathrm{SiO}_{2}-\mathrm{Si}$. The increase in S-parameter due to dynamic vacancy formation is clearly evident.

\section{Electromigration-induced vacancies in $\mathrm{Al}(\mathrm{Cu})$ lines}

The high packing densities of devices in modern integrated circuits raised the issue of the reliability of metallic interconnects. The failure of aluminum and aluminum-alloy interconnects by the formation of voids and hillocks from electromigration has been of immense importance to manufacturers. The mean-time-to-failure often is assessed by noting changes in electrical properties (such as resistance and $1 / f$ noise) and microscopic tests are used to observe structural damages. However, these tests either require sampling many test systems, or they are performed after significant changes have taken place in the interconnect lines. Since material transport along the conductor lines during electromigration is believed to be associated with the generation of vacancies, PAS offers a sensitive probe to observe early stages of this failure mode.

Figure 4 shows an in situ measurement from a system containing $1 \mu \mathrm{m} \mathrm{Al} \mathrm{lines}$ spaced at $1 \mu \mathrm{m}$ on a $\mathrm{SiO}_{2}-\mathrm{Si}$ system. The aluminum lines contained $0.5 \% \mathrm{Cu}$ by weight; $\mathrm{Cu}$ is known to increase mean-time-to-failure. Figure 4 compares the results from a system carrying no current to a system carrying current at $8 \times 10^{4} \mathrm{~A} / \mathrm{cm}^{2}$ and $275^{\circ} \mathrm{C}$. An increase in the S-parameter signal is evident, suggesting dynamic formation of vacancies during current flow. This increase is observed only if the lines' temperature during the current flow is maintained above $100^{\circ} \mathrm{C}$. Elevating the temperature to $275^{\circ} \mathrm{C}$ without applying the current through the lines produced only half that increase, which can be understood in terms of thermal generation of vacancies. Thus, positron results 
showed that vacancies were created in current-carrying lines when their temperature exceeded $100^{\circ} \mathrm{C}$. Similar studies with varying concentrations of solute can give a basic understanding of the role played by the solute atoms in increasing electromigration resistance. An extended positron study is needed to see if these vacancies eventually coalesce into voids leading to line failures.

\section{Conclusion}

This review shows that positron annihilation spectroscopy is invaluable as a probe to reveal the nature, concentration, and spatial distribution of dilute quantities of defects in a few selected systems. With the recent progress in the positron field, this list of applications easily can be extended to include many more systems. The one discussed here were chosen to exemplify the level of sophistication that can be achieved with this technique. In future, with the development of more intense positron beams, depthresolved spectroscopy will be extended to other positron observables, such as lifetime and angular correlation.

\section{Acknowledgements}

We thank our collaborators for many useful discussions. The work was supported by U.S. Department of Energy Under Contract No. DE-AC02-76CH00016.

\section{References}

Alatalo M, Kauppinen H, Saarinen K, Puska M J, Mäkinen J, Hautojärvi P and Nieminen R M 1995 Phys. Rev. BS1 4176

Asoka-Kumar P et al 1993 Phys. Rev. B48 5345

Asoka-Kumar P, Lynn K G and Welch D O 1994a J. Appl. Phys. 764935

Asoka-Kumar P, Stein H J and Lynn K G 1994b Appl. Phys. Lett. 641684

Asoka-Kumar P, Szpala S, Nielsen B, Szeles Cs, Lynn K G, Lanford W A, Shepard C A and Gossmann H -J 1995 Phys. Rev. BS1 4630

Berko S and Erskine J C 1967 Phys. Rev. Lett. 19307

Brandt W and Dupasquier A (eds) 1983 Positron solid state physics, Proc. of int. school of physics, Enrico Fermi, Corse LXXXIII, Varena

Coleman P G and Walker A B (eds) 1986 Proc. of the int. workshop on slow positron beams for solids and surfaces, Norwich, England

Eaglesham D J, Gossmann H -J and Cerullo M 1990 Phys. Rev. Lett. 651127

Gossmann H -J, Schubert E F, Eaglesham D J and Cerullo M 1990 Appl. Phys. Lett. 572440

Gossmann H -J, Asoka-Kumar P, Leung T C, Nielsen B, Lynn K G, Unterwald F C and Feldman L C 1992 Appl. Phys. Lett. 61540

Keeble D J, Umlor M T, Asoka-Kumar P, Lynn K G and Cooke P W 1993 Appl. Phys. Lett. 6387

Khatri R, Asoka-Kumar P, Nielsen B, Roellig L O and Lynn K G 1994 Appl. Phys. Lett. 65330

MacDonald J R, Lynn K G, Boie R A and Robbins M F 1978 Nucl. Instrum. \& Methods 153189

MacKenzie I K, Khoo T L, McDonald A B and McKee B T A 1967 Phys. Rev. Lett. 19946

Nicollian E H and Brews 1982 Metal oxide superconductor, in Physics and Technology (New York: Wiley)

Nielsen B, Lynn K G, Yen C Chen and Welch D O 1987 Appl. Phys. Lett. 511022

Ottewitte E and Weiss A (eds) Slow positron beam techniques for solids and surfaces, Proc. of fifth int. workshop (New York: American Institute of Physics)

Palthingal J C, Asoka-Kumar P, Lynn K G, Posada Y and Wu X Y 1991 Phys. Rev. Lett. 673491

Reichel D C and Yelon W B (eds) 1985 Proc. of the MURR slow positron beam workshop, University of Missouri, Columbia, Missouri (unpublished) 
Schultz P J and Lynn K G 1988 Rev. Mod. Phys. 60701

Schultz P J, Massoumi G R and Simpson P J 1990 Positron beams for solids and surfaces, Proc. of the fourth int. workshop on slow positron beam techniques for solids and surfaces (New York: American Institute of Physics)

Szeles Cs., Asoka-Kumar P, Lynn K G, Gossmann H -J, Unterwald F C and Boone T 1995 Appl. Phys. Lett. 662855

Szpala S, Asoka-Kumar P, Nielsen B, Peng J P, Hayakawa S, Lynn K G and Gossmann H -J 1995 (Unpublished)

Trifthäuser W and Köegel G 1982 Phys. Rev. Lett. 481741

Umlor M T, Asoka-Kumar P, Keeble D J, Cooke P W and Lynn K G 1995 Appl. Surf. Sci. 85295

Vehanen A (ed.) 1984 Slow positrons in surface science, Proc. of the int. workshop, Pajulathi, Finland (Helsinki University of Technology Report No. 135) 